\title{
Clinical and histopathological characteristics of a family with R1141X mutation of pseudoxanthoma elasticum - presymptomatic testing and lack of carrier phenotypes
}

\author{
Gulsen Akoglu ${ }^{\mathrm{I}}$, MD, Qiaoli $\mathrm{Li}^{2}$, PhD, Ozay Gokoz ${ }^{3}$, MD, Ali Serhan Gazyagci ${ }^{4}$, MD, and \\ Jouni Uitto ${ }^{2}, \mathrm{MD}, \mathrm{PhD}$
}

${ }^{1}$ Dermatology Clinic, Ankara Halil Sivgin Cubuk State Hospital, Ankara, Turkey, ${ }^{2}$ Department of Dermatology and Cutaneous Biology, Jefferson Medical College, Philadelphia, PA, USA, ${ }^{3}$ Department of Pathology, Hacettepe University Faculty of Medicine, Ankara, Turkey, and ${ }^{4}$ Ophthalmology Clinic, Ankara Halil Sivgin Cubuk State Hospital, Ankara, Turkey

Correspondence

Gulsen Akoglu, MD

Kardelen M. Baskent Blv. No:224/C/9

Yenimahalle

Ankara

Turkey

E-mail: gusemd@yahoo.com

Gulsen Akoglu and Qiaoli Li contributed equally to this study.

Conflicts of interest: None. Financial disclosure: None.

\begin{abstract}
Background Pseudoxanthoma elasticum (PXE) is a heritable ectopic mineralization disorder affecting cutaneous, ocular, and cardiovascular systems, caused by mutations in the $A B C C 6$ gene. PXE presents with a marked clinical and genetic heterogeneity. Furthermore, heterozygous carriers may present with limited histopathological features. This study was conducted to investigate a patient with PXE and her family members clinically, histopathologically, and genetically.

Methods Clinical and histopathological examinations and mutation analyses of $A B C C 6$ gene were performed.

Results Lesional skin biopsy of the patient with PXE demonstrated clumping and fragmentation of elastic fibers, and calcification in the dermis. Non-lesional axillary skin samples of the husband, daughter, and older son were histopathologically normal. The skin from a similar region of a younger son revealed elastic fibers with some fragmentation and clumping but no mineralization. The patient with PXE was homozygous for the R1141X mutation in the $A B C C 6$ gene. The husband had wild-type alleles, while all children were heterozygous carriers. Daily treatment of antioxidant therapy with tocopherol acetate and ascorbic acid was prescribed to the patient with PXE. After one year, both clinical and histopathological regression of the lesions was observed; however, lesions began to progress during the additional 6-month period of treatment.

Conclusion The mutation analyses of $A B C C 6$ gene are important to determine the genotype of both patients with PXE and putative heterozygous carriers, as histopathological features of carriers may differ even in the same family. The role of antioxidant therapy for PXE is unclear, and there is a need for controlled clinical trials.
\end{abstract}

\section{Introduction}

Pseudoxanthoma elasticum (PXE) is a heritable ectopic mineralization disorder affecting cutaneous, ocular, and cardiovascular systems, caused by mutations in the ABCC6 gene. The characteristic cutaneous findings, yellowish papules or plaques on the lateral sides of the neck and flexural sites of the body, are associated with inelastic, leathery skin. The diagnosis is confirmed by histopathological examination of a skin biopsy demonstrating calcification of pleomorphic elastic fibers. ${ }^{\mathrm{I}-3}$ PXE presents with a marked clinical and genetic, both intra- and interfamilial, heterogeneity. Furthermore, some patients with minimal or absent cutaneous findings and heterozygous
PXE is an autosomal recessively inherited disorder, and the mutated gene, $A B C C 6$, is located on chromosome г 6рг3.г. ${ }^{4,5} \mathrm{Up}$ until now, over 300 distinct mutations in the ABCC6 gene have been defined, the RII4IX mutation being the most common one detected in Caucasians. ${ }^{6}$ The function of the ABCC6 protein has yet to be identified, but it has been proposed that it serves as an efflux transporter and that mutations in the ABCC6 gene lead to deficiency of circulating anti-mineralization factors, thus allowing ectopic mineralization with associated complications to ensue. ${ }^{2,7}$ In this study, a patient with PXE and her family members from the Turkish population were investigated clinically, histopathologically, and genetically for the first time to provide additional insight into PXE. 


\section{Materials and methods}

\section{Clinical evaluation of patients}

A 47-year-old woman presented to the outpatient clinic with cosmetically disturbing skin lesions around her neck and blurred vision. Neither her parents nor she and her husband were consanguineous. She has a 27-year-old daughter and two sons, 10 and 26 years of age. No one except the proband complained about similar skin lesions. Detailed dermatological, physical, ophthalmological, and cardiological examinations were performed on all family members.

\section{Histopathological examinations}

A skin biopsy from the lesional skin of the proband and biopsies from normal-appearing skin of axillary sites of all children and the husband were obtained after informed consent. The specimens were embedded in paraffin and then examined according to routine procedures, in which hematoxylin and eosin, von Kossa, and elastic stains were employed.

\section{ABCC6 gene mutation analysis}

After informed consent, DNA samples for mutation analyses of the $A B C C 6$ gene were performed in all family members. Genomic DNA was isolated from peripheral blood samples (QIAamp Blood Maxi kit; Qiagen Inc., Valencia, CA, USA). Polymerase chain reaction (PCR) was performed using $2.0 \mathrm{U}$ Taq polymerase and $\mathrm{Q}$ buffer (Qiagen), according to the manufacturer's instructions. PCR reactions contained $200 \mathrm{ng}$ DNA as template and $20 \mathrm{ng}$ of each primer in a final volume of $50 \mu$ l. The entire coding region and intron/exon boundaries of $A B C C 6$ were amplified using PCR primers as described previously. ${ }^{6}$ For the detection of the deletion of exons 23-29, the primers described by Le Saux et al. ${ }^{8}$ were used. The PCR products were analyzed with direct sequencing using an Applied Biosystems 3730 Sequencer (Applied Biosystems, Foster City, CA, USA). In the ABCC6 gene, the +1 corresponds to the $A$ nucleotide in the ATG translation initiation codon (GenBank accession no. AF076622).

\section{Results}

\section{Clinical features of the subjects}

The proband had an approximate Io-year history of asymptomatic plaques consisting of multiple yellowish papules, a few millimeters in diameter, over her lateral neck regions and nape (Fig. Ia). She had hypertension and blurred vision for the past two years. Her fundoscopic examination revealed angioid streaks (Fig. Ib). She denied abnormal hemorrhages or claudication. Laboratory tests, including complete blood count, liver and renal function tests, calcium $\times$ phosphate product, lipid profile, and urinalysis were all within normal limits. Fecal occult blood test was negative. Chest x-ray, electrocardiogram, echocardiogram, abdominal ultrasonography, and cranial magnetic resonance imaging did not show abnormal findings. The dermatological, ophthalmological, and cardiovascular examinations, and laboratory tests, echocardiogram, and electrocardiogram evaluations of other family members were all normal, and no angioid streaks were detected.

\section{Histopathology}

Skin biopsy taken from the lateral neck of the proband demonstrated clumping and fragmentation of elastic fibers and calcification in the dermis (Fig. 2a-c). While the biopsy from the skin of the Io-year-old son revealed no mineralization (Fig. 2d,e), elastic fibers with special stain showed some fragmentation and clumping (Fig. 2f). Histopathological examination of skin samples from the non-lesional axillary regions of the husband (not shown), 27-year-old daughter, and 26-year-old son (Fig. 2g-i and j-1) were normal.

\section{Mutations of $A B C C 6$ gene}

The nuclear pedigree of the family is shown in Figure $3 \mathrm{a}$. DNA analysis demonstrated that the proband (I-2) was homozygous for the RII4IX mutation. The husband (I-I) had arginine (R) at the amino acid position II4I in both alleles (wild type), while all children of the proband
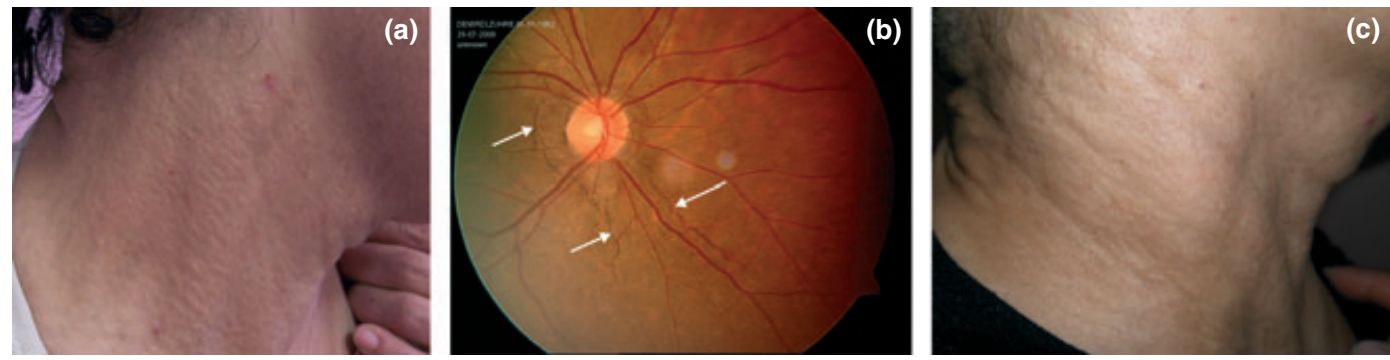

Figure 1 Clinical findings in the family with pseudoxanthoma elasticum (PXE). (a) Plaques of multiple yellowish papules over the neck of the proband with PXE. (b) The patient has angioid streaks (arrows) as observed by fundoscopic examination. (c) Regression of the skin lesions of the patient with PXE after I year duration of antioxidant therapy 

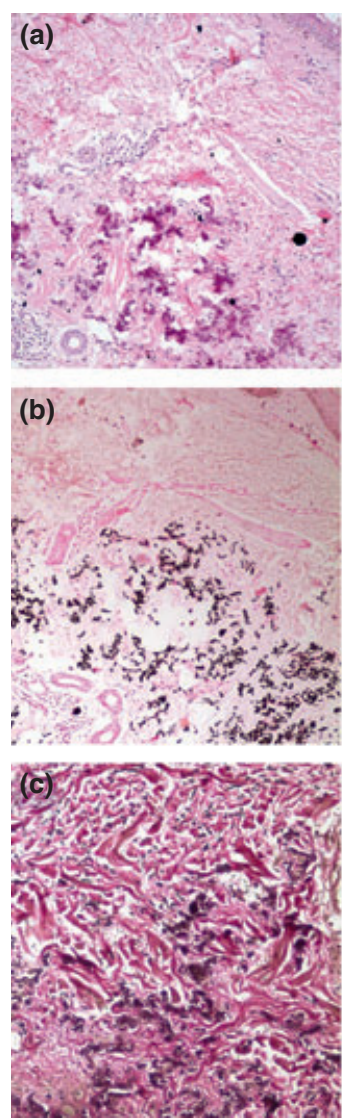
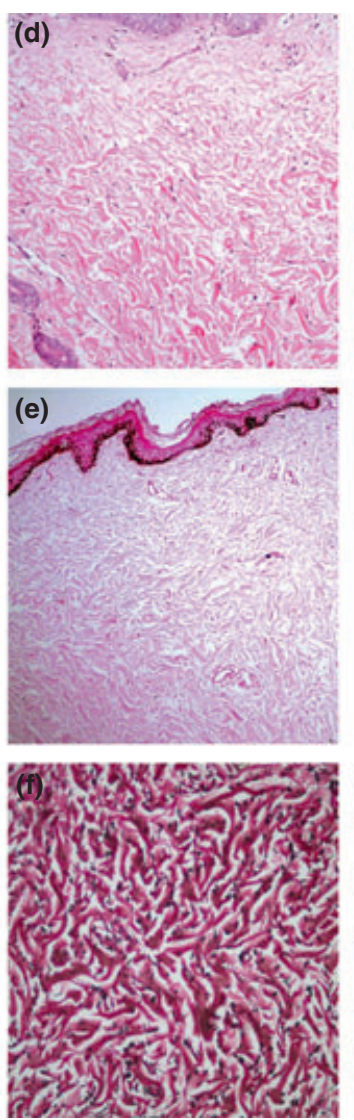
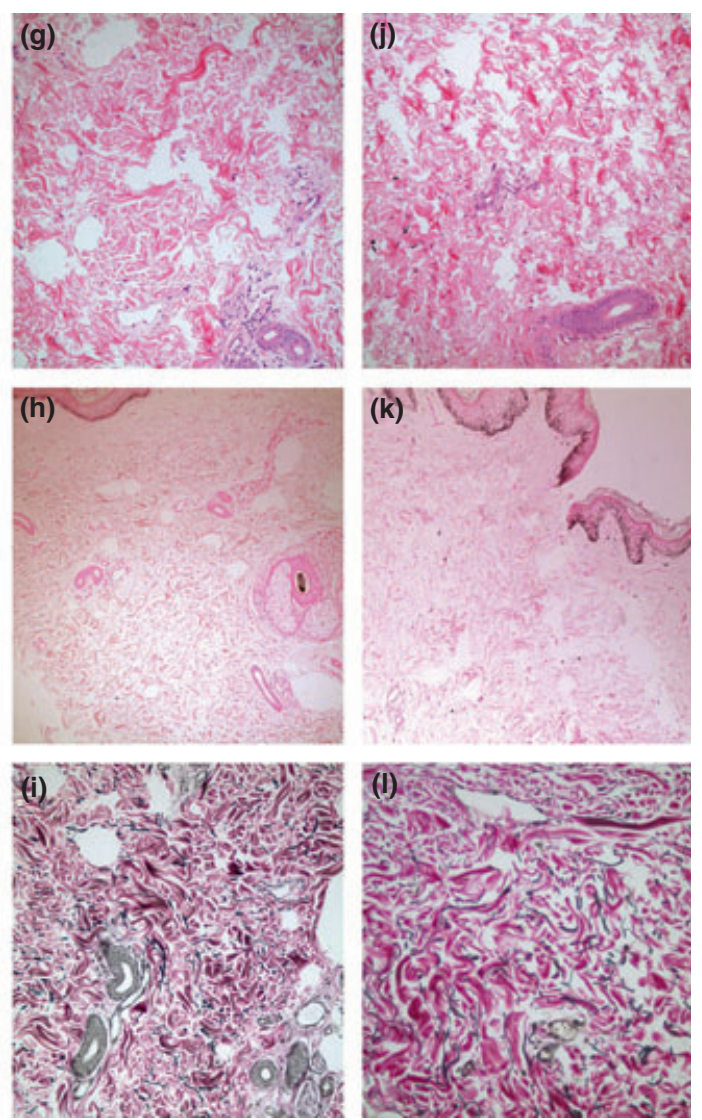

Figure 2 Histopathology of the skin in the proband $(\mathrm{a}-\mathrm{c})$ in the clinically unaffected son who is heterozygous carrier with pathologic elastic fibers $(\mathrm{d}-\mathrm{f})$, and in the normal heterozygous older siblings $(\mathrm{g}-\mathrm{i}, \mathrm{j}-\mathrm{l})$. (a,d,g,j) Hematoxylin and eosin, $\times 200$; $(\mathrm{b}, \mathrm{e}, \mathrm{h}, \mathrm{k})$ von Kossa, $\times$ I००; $(\mathrm{c}, \mathrm{f}, \mathrm{i}, \mathrm{l})$ elastic stain, $\times 200$

were heterozygous carriers for the RII4IX mutation (Fig. 3 b).

Based on clinical, histopathological, and genetic examinations, the proband was diagnosed as having PXE with three major criteria, including characteristic yellowish skin lesions in flexural sites, elastic fiber calcification in lesional skin, and presence of ocular findings. ${ }^{{ }^{\mathrm{T}}}$ The younger son, a carrier of the RII4IX mutation, was considered to have mild histopathological alterations associated with PXE, but he had no evidence of clinical disease. The other children, who were also heterozygous carriers of the RII4IX mutation, had normal histopathology of the skin and no clinical findings suggestive of PXE.

\section{Management of the subjects}

The proband was advised to have a yearly cardiological examination, close monitoring of the eye complications, avoidance of nonsteroidal anti-inflammatory drugs and warfarin, and estrogens and hormone replacement therapy. She was recommended to exercise regularly without contact sport, control her weight, and avoid smoking. In addition to these recommendations, daily treatment with I50 IU of tocopherol acetate and I000 $\mathrm{mg}$ of ascorbic acid was prescribed. Within the first year of the follow-up period, papules appeared to regress (Fig. Ic), and clinically there was no significant further deterioration of fundoscopic findings. During treatment, all laboratory tests remained within normal limits, and no adverse effects were noted.

To determine the histopathological response to the above therapy, a second biopsy specimen was taken from the lesional skin, just near the scar of the first biopsy, one year after therapy was initiated. The histopathological examination suggested less clumping and fragmentation of elastic fibers and calcification in the dermis (Fig. 4a,b). However, no improvement was detected in fundoscopic examination and vision. The patient continued therapy for an additional year, but she subsequently discontinued treatment as the skin lesions were found to progress again during the last six months of therapy. 
(a)

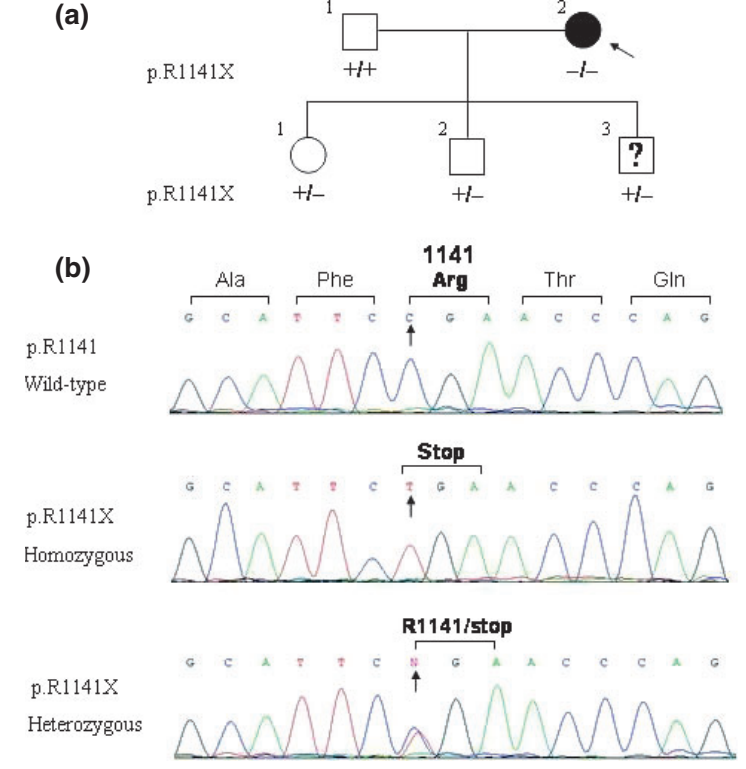

Figure 3 Family pedigree (a), and mutation analysis demonstrating the presence of $R_{I}$ I I I $_{X}$ mutation (b). The proband ( $\mathrm{I}-2$; arrow) is homozygous for the mutation, while the husband of the proband (I-I) shows wild-type sequence, RII4I. The three children (II-I, 2,3) are heterozygous carriers of the mutation

\section{Discussion}

The current PXE classification categorizes patients depending on clinical and histopathological findings (Tables I and 2). ${ }^{\mathrm{I}}$ According to this classification scheme, the proband in our study was in category I with three major features. The two older children had normal elastic fibers without calcification; similar to the histopathological features in the father who had the wild-type ABCC6 gene and detection of only one mutant allele established that they were heterozygous, clinically unaffected carriers of PXE. These findings point to the importance of presymptomatic testing in families of patients with PXE as there is a lack of carrier phenotypes. The youngest child, a Io-year-old boy, had fragmented and clumped elastic fibers without calcification. It has been suggested that abnormally structured elastic fibers without calcification may be seen at the early stages of disease or may be a feature of heterozygous patients. ${ }^{9-\mathrm{II}}$ Until now, however, there have been only a few studies investigating the histopathological characteristics of skin in heterozygotes. ${ }^{9, \mathrm{II}, \mathrm{I} 2}$ Before the identification of ABCC6 as the mutated gene in PXE, the study by Bacchelli et al. ${ }^{9}$ found increased elastin and elastic fiber polymorphism in I8 asymptomatic, putative heterozygote family members as compared to healthy controls, but the changes were less pronounced
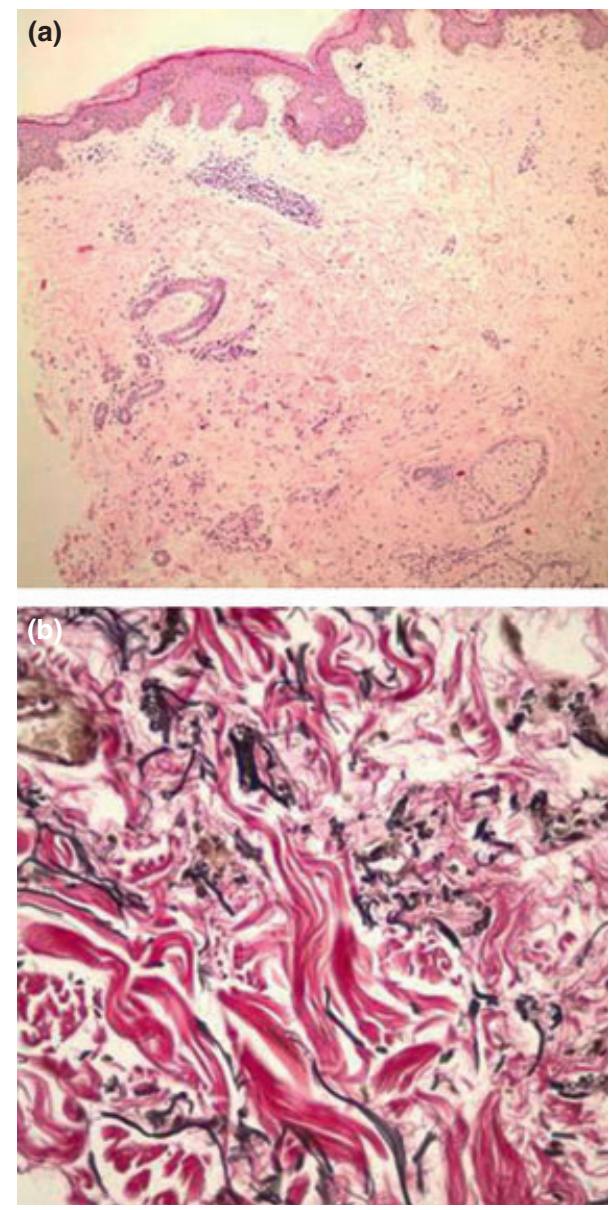

Figure 4 Less calcification (a) and elastic fibers (b) in the second biopsy of first patient, when compared with the first biopsy. (a) Hematoxylin and eosin, × $\times 400$

Table 1 Criteria for the diagnosis of pseudoxanthoma elasticum

\section{Major criteria}

1. Characteristic skin involvement

2. Characteristic histopathologic features of lesional skin

3. Characteristic ocular disease in adults older than 20 years of age

\section{Minor criteria}

1. Characteristic histopathologic features of nonlesional skin

2. Family history of pseudoxanthoma elasticum in first-degree relatives

than in patients with PXE. A few of these individuals also had areas of mild mineralization. Martin et al. ${ }^{\text {II }}$ examined skin biopsies of seven relatives of patients with PXE confirmed to be heterozygotes by mutation analysis of $A B C C 6$. In these patients, increased amounts of short, thick, fragmented elastic fibers were detected. Besides, 
Table 2 Classification of $\mathrm{PXE}^{\mathrm{a}}$

\begin{tabular}{|c|c|c|c|c|}
\hline $\begin{array}{l}\text { Category I ( } 3 \text { major } \\
\text { criteria) }\end{array}$ & $\begin{array}{l}\text { Category lla ( } 1 \text { major }+ \\
2 \text { minor criteria) }\end{array}$ & $\begin{array}{l}\text { Category Ilb ( } 1 \text { major }+ \\
1 \text { minor criteria) }\end{array}$ & $\begin{array}{l}\text { Category Ilc ( } 1 \text { major }+ \\
1 \text { minor criteria) }\end{array}$ & Category IId (2 minor criteria) \\
\hline $\begin{array}{l}\text { 1. Characteristic yellow } \\
\text { skin lesions in flexural } \\
\text { sites }\end{array}$ & 1. Angioid streaks & 1. Angioid streaks & 1. Angioid streaks & $\begin{array}{l}\text { 1. Family history of PXE in first- } \\
\text { degree relatives }\end{array}$ \\
\hline $\begin{array}{l}\text { 2. Elastic fiber calcification } \\
\text { in lesional skin } \\
\text { 3. Ocular disease in adults }\end{array}$ & $\begin{array}{l}\text { 2. Elastic fiber calcification } \\
\text { in nonlesional skin } \\
\text { 3. Family history of PXE in } \\
\text { first-degree relatives }\end{array}$ & $\begin{array}{l}\text { 2. Elastic fiber calcification } \\
\text { in nonlesional skin }\end{array}$ & $\begin{array}{l}\text { 2. Family history of PXE } \\
\text { in first-degree relatives }\end{array}$ & $\begin{array}{l}\text { 2. Elastic fiber calcification } \\
\text { in nonlesional skin }\end{array}$ \\
\hline
\end{tabular}

PXE, pseudoxanthoma elasticum.

adapted from Ref.I.

foci of abnormal elastic tissue mineralization were also found. The abnormal elastic fiber morphology in carriers of a single ABCC6 mutation was suggestive of having a phenotype midway between the wild type and homozygote skin. Subsequently, Plomp et al. ${ }^{\mathrm{I} 2}$ reported detailed analyses of I 5 homozygous and 4I heterozygous cases, and $\mathrm{I} 2$ healthy controls. Increased elastin was reported in $63 \%$ and fragmentation of elastic fibers in $57 \%$ of heterozygous individuals' skin, while no clumping or calcification was seen. The percentages of these findings were higher in homozygous cases. Upon these findings, the authors suggested that an increase and fragmentation of elastin in the skin of heterozygotes might not be a reliable criterion to differentiate them from homozygous patients. Although our study includes only three heterozygous carriers, the data may add information to our knowledge about PXE. Specifically, the histopathologic features in one of the carriers in our study revealed short, thick, fragmented elastic fibers without mineralization, findings consistent with previous studies. It remains to be investigated why only one of the three heterozygous carriers demonstrated pathology of elastic fibers and the others did not. One reason may be the site selection for normal appearing skin. The axillary region is generally preferred for this kind of investigation in PXE, as biopsy from this site of skin is cosmetically acceptable, and it is not affected by external factors, such as ultraviolet radiation that alters the elastic fibers in the dermis. ${ }^{13}$ Nevertheless, future studies investigating other sites, such as the inguinal area, may help to diagnose heterozygous carriers, in which skin biopsy may be a diagnostic tool to obtain clues of PXE.

The current classification system of PXE is based on major and minor criteria that describe skin findings, histopathology with calcification and fragmentation of elastic fibers, family history of PXE in first-degree relatives, and presence of angioid streaks (Tables I and 2). ${ }^{\mathrm{I}}$ Category I is considered to represent definitive PXE. However, the clinical evaluation of an individual may not always allow the diagnosis to be made, as the expression of PXE phenotype is of late onset and often not present in younger individuals. ${ }^{\mathrm{I}, 2}$ Typical skin and eye findings are usually noted in adulthood, while angioid streaks are rarely detected before the age of IO. $^{\text {I2 }}$ Histopathological findings may also vary from one patient to another, together with variable clinical findings. ${ }^{\mathrm{I}, 9, \mathrm{II}, \mathrm{I} 2}$ Elastic fiber calcification of the non-lesional skin is one of the minor criteria; on the other hand, calcification may not be demonstrable in about $20 \%$ of patients with PXE. ${ }^{\mathrm{I} 2}$ Category II represents patients with tentative diagnosis, but it is often unclear whether they are heterozygous carriers or they will develop typical findings of PXE later in life. ${ }^{\mathrm{I}}$ This uncertainty of the value of histopathological changes in the skin in heterozygous carriers emphasizes the importance of DNA-based mutation analysis in the ABCC6 gene. It should be noted, however, that the most commonly employed mutation detection strategies that amplify exons and flanking intronic sequences of the ABCC6 gene from genomic DNA may not detect all mutations, including those in the regulatory regions or in intronic sequences away from intron-exons junctions, or large allelic deletions. ${ }^{6,8}$

Early identification and accurate diagnosis of PXE, homozygous or compound heterozygous for ABCC6 mutations, as well as of the heterozygous carriers, is important for several reasons. First, accurate and early diagnosis of definitive PXE allows institution of appropriate monitoring of complications and measures to counteract the development of the disease. Secondly, while the heterozygous carriers do not develop the characteristic cutaneous findings of PXE, carriers of ABCC6 mutations, particularly the RII4IX, have been suggested to be at risk for premature coronary artery disease. ${ }^{\mathrm{I} 3, \mathrm{I} 4}$ These considerations are emphasized by the phenotypic heterogeneity that is noted in patients with PXE, even within the same family. This phenotypic variability has been suggested to reflect the influence of genetic modifier genes as 
well as the lifestyle and dietary variables in these patients.

It is clear that mutations in the $A B C C 6$ gene underlie PXE in the classic form of PXE; however, the pathomechanistic details leading from ABCC6 mutations into peripheral ectopic mineralization of connective tissues remain unclear. ${ }^{3}$ The role of oxidative stress has been suggested to play a role in modifying the PXE phenotype. For example, intracellular reactive oxygen species have been reported to be high in fibroblasts cultured from the skin of patients with PXE, and the total antioxidant status was lower in PXE fibroblasts as compared to controls. ${ }^{15,{ }^{16}}$ In this context, Zarbock et al. ${ }^{17}$ have detected polymorphisms in antioxidant genes associated with altered enzyme activity of CAT, GPXI, and $\mathrm{SOD}_{2}$ in patients with early clinical manifestation of the symptoms. Addition of vitamin $\mathrm{E}$ to fibroblasts from patients with PXE has been shown to reverse the oxidative stress. ${ }^{\text {I6 }}$ Based on these and similar observations, it has been suggested that antioxidant therapy might improve the presentation of patients with PXE, and a study by Takata et al. ${ }^{\mathrm{I} 8}$ has suggested that administration of tocopherol and ascorbic acid may improve the appearance of skin lesions in PXE. Depending on these observations, we administered the proband antioxidant therapy consisting of $\mathrm{I} 50 \mathrm{IU}$ of tocopherol and I $\mathrm{g}$ of ascorbic acid daily. It appeared that skin lesions may have regressed over the initial I8-month period of treatment, but thereafter the lesions appeared to progress again. These outcomes may suggest that antioxidant therapy could suppress the oxidant microenvironment of the skin for a certain time but may not be effective for long-term maintenance. Furthermore, it is not clear as to the optimal dose of these agents. It should also be noted that the treatment of $A b c c 6^{-1-}$ mice, an animal model of PXE, failed to counteract the mineralization. ${ }^{\text {I9 }}$ Specifically, although it was shown that these mice suffered from chronic oxidative stress, diet including vitamins $\mathrm{C}$ and $\mathrm{E}$, selenium, and $\mathrm{N}$-acetylcysteine did not prevent progression of the ectopic mineralization. Thus, the role of antioxidant therapy for PXE is unclear, and there is a need for controlled clinical trials for patients with PXE.

Quite recent studies have demonstrated the effectiveness of oral phosphate binders and diet enriched in magnesium in counteracting the calcium deposition in PXE mice. ${ }^{20-22}$ Specifically, supplementation of the normal mouse diet with fivefold higher amount of magnesium completely prevented the mineralization but did not reverse the existing mineral deposits. Preliminary clinical trials have also suggested that phosphate binders and magnesium may be helpful for treatment of patients with PXE. ${ }^{23,24}$ These observations, if applicable to larger cohorts of patients in future clinical trials, would suggest that such treatment should be considered for PXE and should be initiated as soon as the diagnosis has been established.

\section{Acknowledgments}

Special thanks to the patient and her family members for participation in the study.

\section{References}

I Lebwohl M, Neldner K, Pope FM, et al. Classification of pseudoxanthoma elasticum: report of a consensus conference. J Am Acad Dermatol I994; 30: I03-I07.

2 Uitto J, Li Q, Jiang Q. Pseudoxanthoma elasticum: molecular genetics and putative pathomechanisms. J Invest Dermatol 2010; I30: 66I-670.

3 Uitto J, Bercovitch L, Terry SF, et al. Pseudoxanthoma elasticum: progress in diagnostics and research towards treatment: summary of the 2010 PXE International Research Meeting. Am J Med Genet A 20I I; I55A: I 5 I7-I 526.

4 Bergen AA, Plomp AS, Schuurman EJ, et al. Mutations in ABCC6 cause pseudoxanthoma elasticum. Nat Genet 2000; 25: 228-23I.

5 Ringpfeil F, Lebwohl MG, Christiano AM, et al. Pseudoxanthoma elasticum: mutations in the MRP6 gene encoding a transmembrane ATP-binding cassette (ABC) transporter. Proc Natl Acad Sci USA 2000; 97: 600I6006.

6 Pfendner EG, Vanakker O, Terry SF, et al. Mutation detection in the ABCC6 gene and genotype-phenotype analysis in a large international case series affected by pseudoxanthoma elasticum. J Med Genet 2007; 44: $62 \mathrm{I}-628$.

7 Jiang Q, Endo M, Dibra F, et al. Pseudoxanthoma elasticum is a metabolic disease. J Invest Dermatol 2009; I29: $348-354$.

8 Le Saux O, Beck K, Sachsinger C, et al. A spectrum of ABCC6 mutations is responsible for pseudoxanthoma elasticum. Am J Hum Genet 2001; 69: 749-764.

9 Bacchelli B, Quaglino D, Gheduzzi D, et al. Identification of heterozygote carriers in families with a recessive form of pseudoxanthoma elasticum (PXE). Mod Pathol I999; I2: III2-II23.

Io Basalyga DM, Simionescu DT, Xiong W, et al. Elastin degradation and calcification in an abdominal aorta injury model: role of matrix metalloproteinases. Circulation 2004; I I0: 3480-3487.

I I Martin L, Chassaing N, Delaite D, et al. Histological skin changes in heterozygote carriers of mutations in ABCC6, the gene causing pseudoxanthoma elasticum. J Eur Acad Dermatol Venereol 2007; 21: 368 373 .

I 2 Plomp AS, Toonstra J, Bergen AA, et al. Proposal for updating the pseudoxanthoma elasticum classification system and a review of the clinical findings. Am J Med Genet A 2010; I52A: 1049-1058. 
I3 Trip MD, Smulders YM, Wegman JJ, et al. Frequent mutation in the ABCC6 gene (RII4IX) is associated with a strong increase in the prevalence of coronary artery disease. Circulation 2002; I06: 773-775.

I4 Köblös G, Andrikovics H, Prohászka Z, et al. The RII4IX loss-of-function mutation of the ABCC6 gene is a strong genetic risk factor for coronary artery disease. Genet Test Mol Biomarkers 2010; I4: 75-78.

I 5 Boraldi F, Annovi G, Guerra D, et al. Fibroblast protein profile analysis highlights the role of oxidative stress and vitamin $\mathrm{K}$ recycling in the pathogenesis of pseudoxanthoma elasticum. Proteomics Clin Appl 2009; 3: $1084-1098$.

I6 Pasquali-Ronchetti I, Garcia-Fernandez MI, Boraldi F, et al. Oxidative stress in fibroblasts from patients with pseudoxanthoma elasticum: possible role in the pathogenesis of clinical manifestations. J Pathol 2006; 208: 54-6I.

I7 Zarbock R, Hendig D, Szliska C, et al. Pseudoxanthoma elasticum: genetic variations in antioxidant genes are risk factors for early disease onset. Clin Chem 2007; 53: I734-I740.

I 8 Takata T, Ikeda M, Kodama H, et al. Treatment of pseudoxanthoma elasticum with tocopherol acetate and ascorbic acid. Pediatr Dermatol 2007; 24: $424-425$.

I9 Li Q, Jiang Q, Uitto J. Pseudoxanthoma elasticum: oxidative stress and antioxidant diet in a mouse model (Abcc6-/-). J Invest Dermatol 2008; I28: I I60-I I64.

20 LaRusso J, Jiang Q, Li Q, et al. Ectopic mineralization of connective tissue in Abcc6-/- mice: effects of dietary modifications and a phosphate binder-a preliminary study. Exp Dermatol 2008; I7: 203-207.

2I Li Q, Larusso J, Grand-Pierre AE, et al. Magnesium carbonate-containing phosphate binder prevents connective tissue mineralization in Abcc6(-/-) micepotential for treatment of pseudoxanthoma elasticum. Clin Transl Sci 2009; 2: 398-404.

22 LaRusso J, Li Q, Uitto J. Elevated dietary magnesium prevents tissue mineralization in a mouse model of pseudoxanthoma elasticum (Abcc6(-/-)). J Invest Dermatol 2009; I29: I388-I394.

23 Sherer DW, Singer G, Uribarri J, et al. Oral phosphate binders in the treatment of pseudoxanthoma elasticum. J Am Acad Dermatol 2005; 53: 6IO-6I 5 .

24 Yoo JY, Blum RR, Singer GK, et al. A randomized controlled trial of oral phosphate binders in the treatment of pseudoxanthoma elasticum. J Am Acad Dermatol 2OII; 65: 34I-348. 